\title{
Acute Kidney Injury Caused by TAFRO Syndrome in a Chinese Patient: Efficacy of Long-Term Corticosteroids Combined with Bortezomib and Cyclophosphamide
}

\author{
Peng Xia ${ }^{a}$ Lu Zhang $^{b}$ Menglian Zou ${ }^{c}$ Tengyue Zhang ${ }^{c}$ Ran $\mathrm{Li}^{\mathrm{c}}$ \\ Xiaoxiao Shic Jing Wang ${ }^{d}$ Yan Qin ${ }^{a}$ Xuemei Li ${ }^{a}$ Jian Li $^{b}$ \\ Limeng Chen ${ }^{\mathrm{a}}$ \\ a Department of Nephrology, Peking Union Medical College Hospital, Chinese Academy \\ of Medical Sciences, Beijing, China; ${ }^{b}$ Department of Hematology, Peking Union Medical \\ College Hospital, Chinese Academy of Medical Sciences, Beijing, China; ${ }^{\mathrm{C}}$ Department of \\ Internal Medicine, Peking Union Medical College Hospital, Chinese Academy of Medical

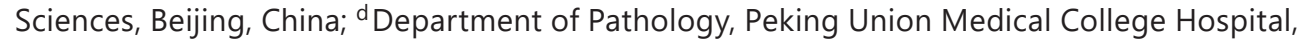 \\ Chinese Academy of Medical Sciences, Beijing, China
}

\section{Keywords}

Acute kidney injury · TAFRO syndrome · Bortezomib

\begin{abstract}
Introduction: Thrombocytopenia, ascites, myelofibrosis, renal dysfunction, and organomegaly (TAFRO) syndrome is a newly recognized and rare clinical subtype of Castleman disease. Renal involvement in TAFRO syndrome usually presents with mild proteinuria, microscopic hematuria, and acute renal injury requiring temporary renal replacement. There is no standard therapy available and treatment failures are common, leading to a poor prognosis. We report a case of acute renal failure caused by TAFRO syndrome, successfully managed by long-term corticosteroids combined with bortezomib and cyclophosphamide. Case Presentation: The patient was a 52-year-old female who presented with fever, anasarca, oliguria, and abdominal distension at first. She progressed rapidly to anuric renal failure requiring hemodialysis. She also demonstrated thrombocytopenia, anemia, coagulopathy, and a hyperinflammatory status. Her CT scan showed severe polyserositis, splenomegaly, and lymphadenopathy. Her serum vascular epithelial growth factor level was significantly elevated. Axillary lymph node biopsy showed hyaline-vascular type Castleman disease, supporting the diagnosis of TAFRO
\end{abstract}

Peng Xia and Lu Zhang contributed equally to this work. 


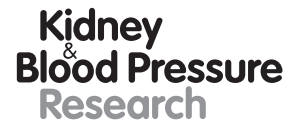

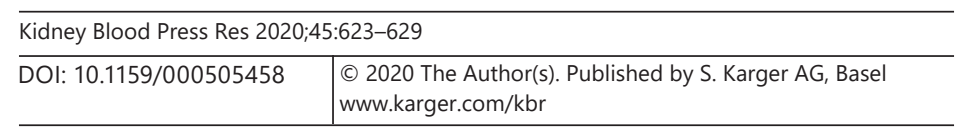

Xia et al.: Acute Kidney Injury Caused by TAFRO Syndrome

syndrome. Her renal function recovered after high-dose steroids and supportive treatment. A weekly dosing regimen of bortezomib, cyclophosphamide, and dexamethasone combined with medium dose prednisone in between were deployed. Her blood cell count and renal function remained stable after 6 months. The inflammation was suppressed and the polyserositis resolved completely. Conclusion: TAFRO syndrome is rare and has a poor prognosis due to the lack of standard treatment. Our patient might be the first TAFRO case successfully treated by bortezomib, cyclophosphamide, and corticosteroids.

(C) 2020 The Author(s)

Published by S. Karger AG, Basel

\section{Introduction}

Thrombocytopenia, ascites, myelofibrosis, renal dysfunction, and organomegaly (TAFRO) syndrome is a newly recognized and rare clinical entity reported firstly in 2010 by Takai et al. [1]. It is considered as a sub-type of human herpes virus 8 (HHV-8)-negative idiopathic multicentric Castleman's disease (iMCD) that shares common cytokine abnormalities such as elevated interleukin (IL)-6 [2]. Renal involvement in TAFRO syndrome is common, and patients present with proteinuria, microscopic hematuria, and acute renal injury requiring temporary renal replacement [3]. A recent case series showed a mean serum creatinine level of $132.6 \mu \mathrm{mol}(39.8-219.2)$ in 8 patients [4]. There is no standard therapy for this disease. Despite multiple treatment options including corticosteroids, immunosuppressive reagents, rituximab, and anti-IL-6 treatment, TAFRO syndrome has a poor prognosis [5-7]. We report a case of TAFRO syndrome with acute renal injury; the patient responded well to weekly bortezomib, cyclophosphamide treatment, and longterm corticosteroids.

\section{Case Presentation}

The patient was a 52-year-old female with an unremarkable medical history. She presented on 4 January 2019 with fever (approx. $38.0^{\circ} \mathrm{C}$ ) accompanied by wheal erythema on both arms, anasarca, and abdominal distension. Her estimated urine output was $<400 \mathrm{~mL} /$ day. Initial laboratory tests at the local hospital showed anemia (hemoglobin, Hb 94 g/L), thrombocytopenia (platelet count, PLT $71 \times 10^{9} / \mathrm{L}$ ), hypoalbuminemia (albumin, ALB 21 $\mathrm{g} / \mathrm{L}$ ), and renal dysfunction (serum creatinine, $\mathrm{s} C \mathrm{Cr} 1.9 \mathrm{mg} / \mathrm{dL}$; estimated glomerular filtration rate, eGFR $29.1 \mathrm{~mL} / \mathrm{min} / 1.73 \mathrm{~m}^{2}$ ). Her urine dipstick showed $2+$ for protein and $1+$ for blood.

The symptoms of fever and rash diminished after 10 days of glucocorticoid treatment (dose unknown). However, after discontinuation of the glucocorticoids, her edema and abdominal distension worsened. She developed anuria, shortness of breath, and paroxysmal nocturnal dyspnea in early February. Her labs in our hospital showed hypoxemia $\mathrm{PaO}_{2} 72$ $\mathrm{mm} \mathrm{Hg} ; \mathrm{SaO}_{2} 89 \%$ ), progressive renal failure (sCr $7.2 \mathrm{mg} / \mathrm{dL}$; eGFR $5.9 \mathrm{~mL} / \mathrm{min} / 1.73 \mathrm{~m}^{2}$; urea $155.5 \mathrm{mg} / \mathrm{dL}$ ) complicated with hyperkalemia (K $6.1 \mathrm{mmol} / \mathrm{L}$ ), mild proteinuria (protein/ creatinine ratio $710 \mathrm{mg} / \mathrm{g} \mathrm{Cr}$ ), severe anemia (Hb $62 \mathrm{~g} / \mathrm{L}$ ), and thrombocytopenia (PLT $78 \times$ $10^{9} / \mathrm{L}$ ), without schistocytes. Other laboratory abnormalities included coagulopathy and a hyperinflammatory status (C-reactive protein, CRP $249.27 \mathrm{mg} / \mathrm{L}$; IL-6, $6.7 \mathrm{pg} / \mathrm{mL}$; erythrocyte sedimentation rate, ESR $>140 \mathrm{~mm} / \mathrm{h}$ ). Her alkaline phosphatase (ALP) was elevated (254 U/L) while transaminase and bilirubin were normal (online suppl. Table S1; for all online suppl. material, see www.karger.com/doi/10.1159/000505458). Antinuclear antibodies, antineutrophil cytoplasmic antibodies, and anti-glomerular basement membrane (anti-GBM) antibodies were all negative. The CT scan showed bilateral pleural effusion, peri- 
Kidney

Blood Pressure

Research
Kidney Blood Press Res 2020;45:623-629
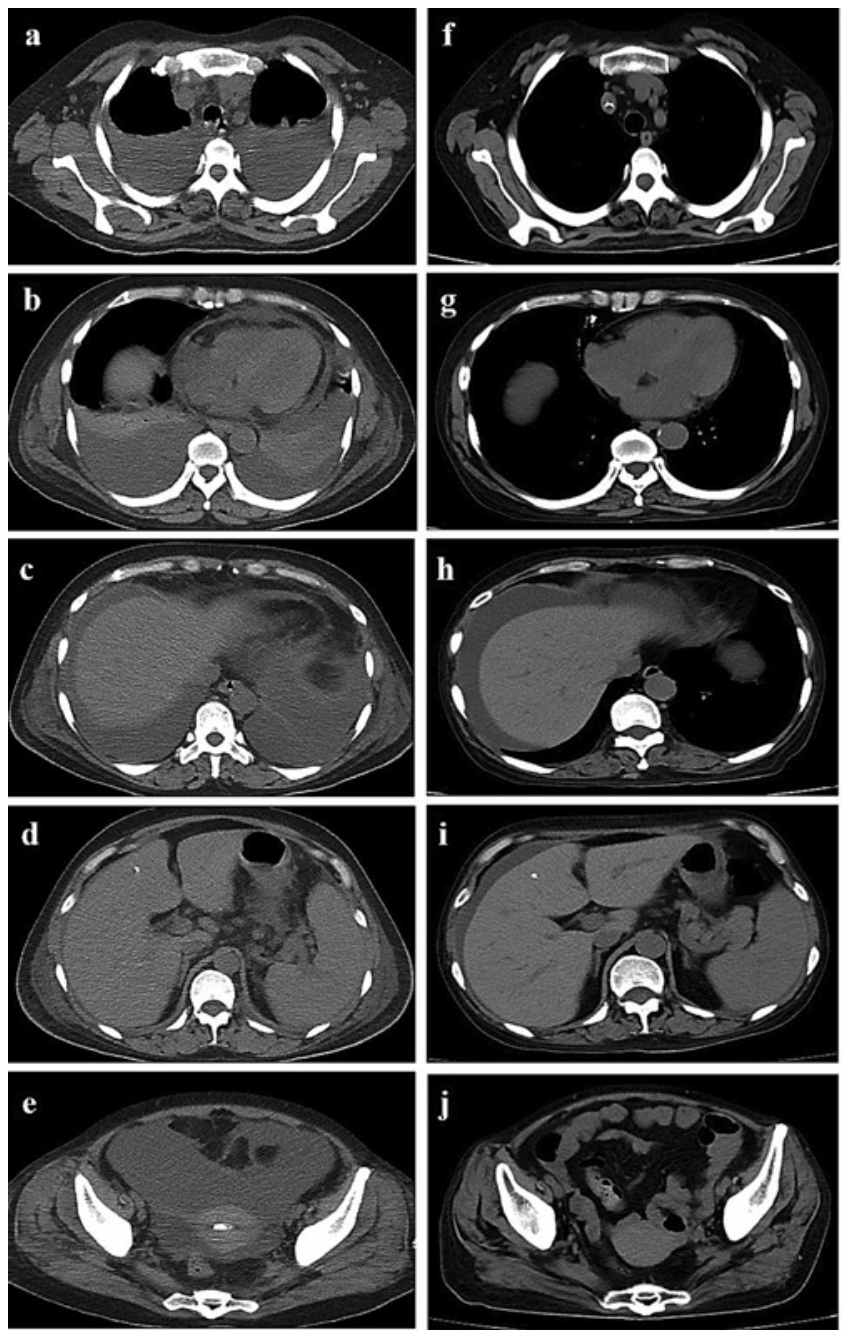

Feb. $13^{\text {th }}, 2019$
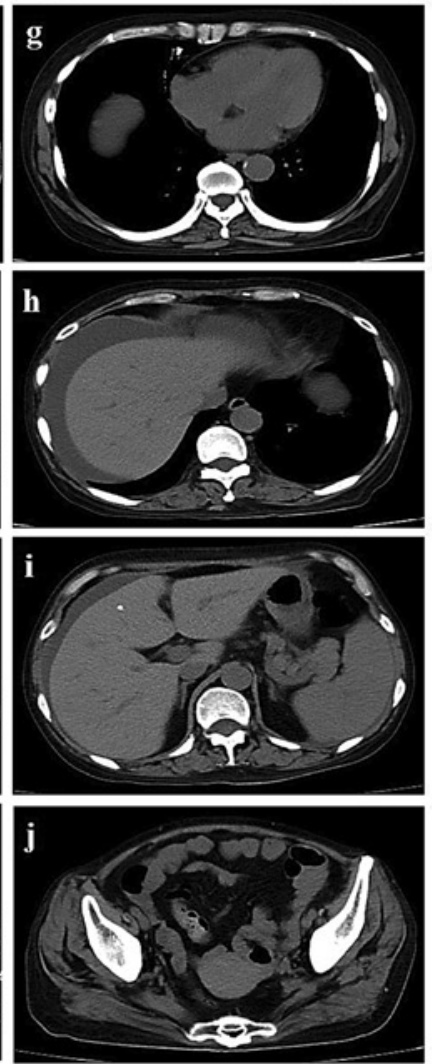

May. $7^{\text {th }}, 2019$
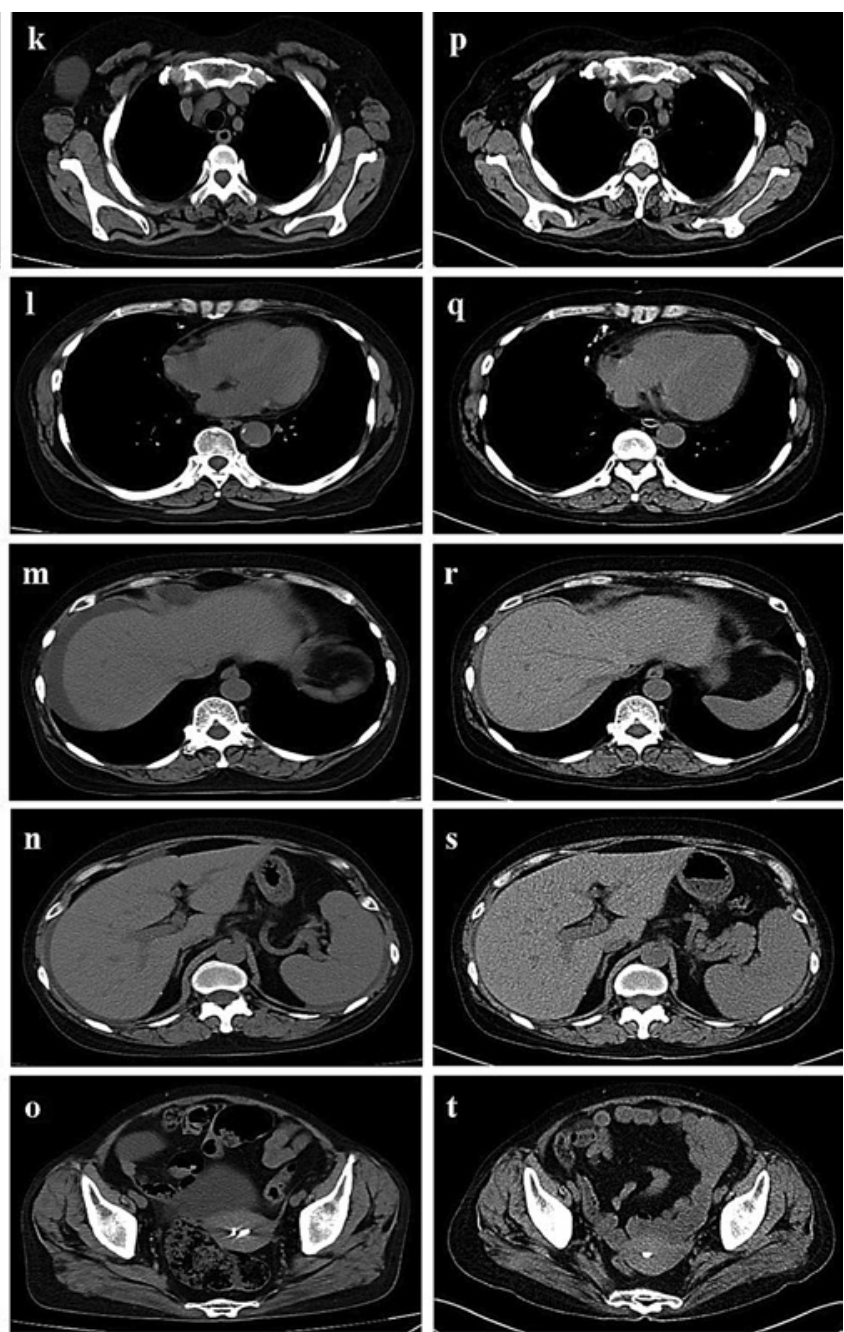

Aug. 13 ${ }^{\text {th }}, 2019$
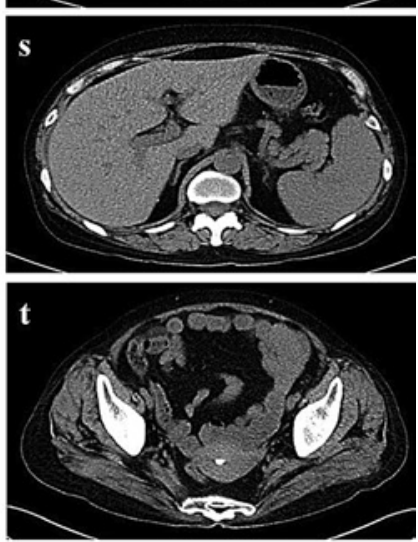

Oct. $9^{\text {th }}, 2019$

Fig. 1. The patient responded well to steroids combined with bortezomib, cyclophosphamide, and dexamethasone (BCD). Initially, the patient presented with bilateral pleural effusion, pericardial effusion, and ascites (a-c, e), splenomegaly (d), and lymphadenopathy (a). After 2 months of high-dose steroids (prednisone $>50 \mathrm{mg}$ /day on average) and 1 month of bortezomib and cyclophosphamide, her pleural effusion, pericardial effusion, and ascites were well controlled ( $\mathbf{f}-\mathbf{j})$, and her splenomegaly improved $(\mathbf{j})$. We continued weekly BCD dosing and tapered the steroids in between to prednisone $5 \mathrm{mg} /$ day for the next $5 \mathrm{months}$. She remained stable clinically, showing complete remission of the polyserositis and continued improvement of the splenomegaly on the CT scan $(\mathbf{k}-\mathbf{t})$.

cardial and abdominal effusion, splenomegaly, and multiple enlarged lymph nodes in the cervical and axillary regions (Fig. 1). After 12 days of corticosteroid therapy (methylprednisolone $80 \mathrm{mg} /$ day) and supportive treatment including one 8-h session of continuous venovenous hemofiltration using citrate anticoagulation and six sessions of 3-4 h of intermittent hemodialysis using unfractionated heparin anticoagulation, pleural effusions, ascites drainage, and blood transfusion, her renal function fully recovered $(\mathrm{sCr} 0.8 \mathrm{mg} / \mathrm{dL}$ and eGFR $84.4 \mathrm{~mL} / \mathrm{min} / 1.73 \mathrm{~m}^{2}$ ). Her urine output increased to $800-1,300 \mathrm{~mL} /$ day. The anemia, thrombocytopenia, and coagulopathy were corrected. Her polyserositis also improved significantly. Most of the enlarged lymph nodes shrank. The 24-h urine protein at this time was $1.8 \mathrm{~g}$, and the urine sediment showed only mild glomerular hematuria. 


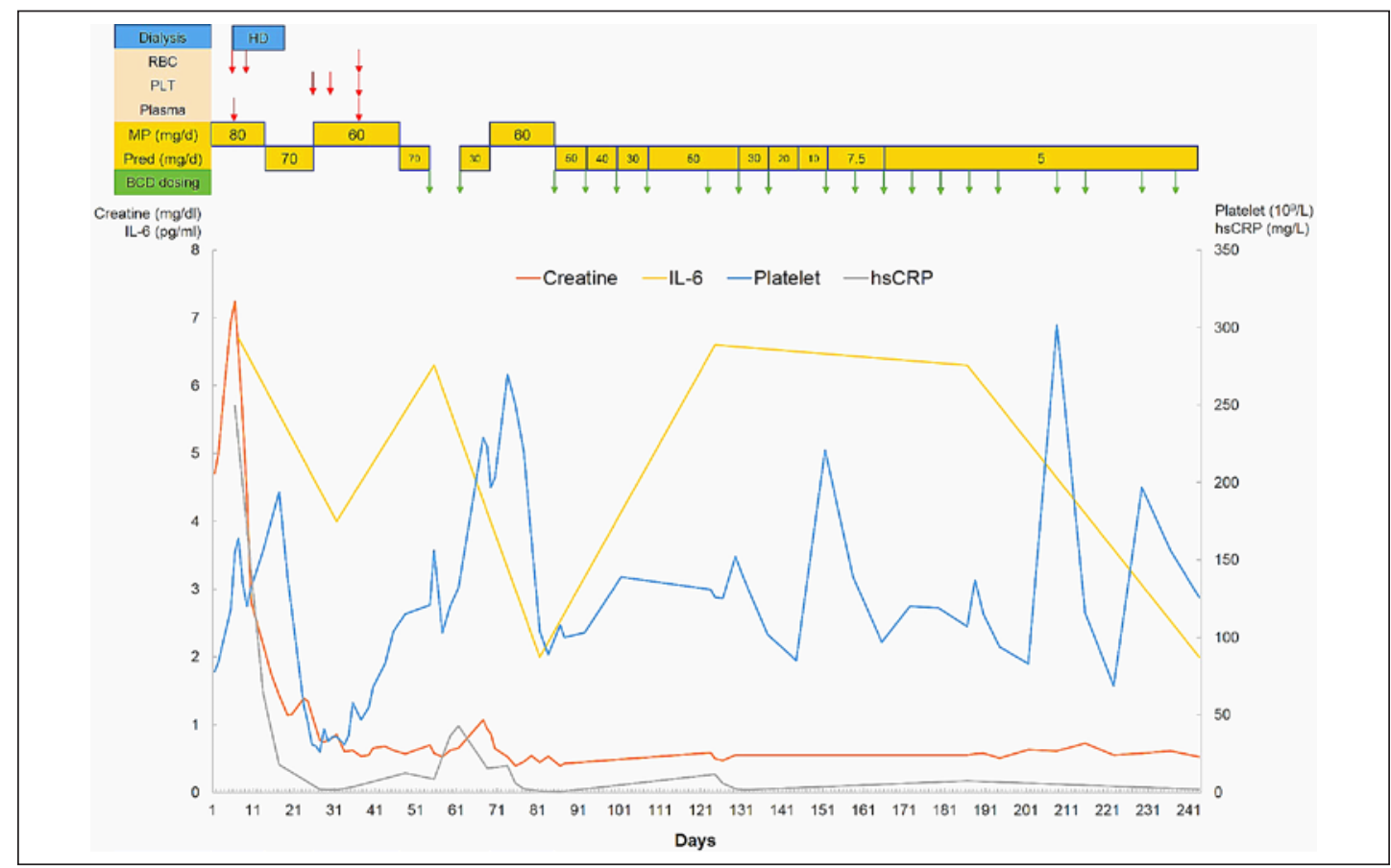

Fig. 2. The patient had a good response to steroids combined with weekly dosing of bortezomib, cyclophosphamide, and dexamethasone (BCD). She required frequent blood transfusion and hemodialysis initially. Her renal function and platelet count recovered after high-dose methylprednisolone treatment and remained stable under weekly BCD dosing and tapering prednisone in between. Inflammatory markers including high-sensitivity C-reactive protein (hsCRP) and interleukin (IL)-6 were suppressed. The patient showed temporary mild myelosuppression after the treatment, which would recover quickly by leaving out 1 dose of BCD. MP, methylprednisolone; Pred, prednisone; HD, hemodialysis; RBC, red blood cell transfusion; PLT, platelet transfusion.

However, right after we tapered the steroids to prednisone $70 \mathrm{mg} /$ day, her ascites relapsed and led to bowel obstruction (online suppl. Fig. S1). The PLT dropped to $26 \times 10^{9} / \mathrm{L}$ within 5 days. Meanwhile, she developed hospital-acquired pneumonia that required intravenous antibiotics. On 7 March, her serum vascular epithelial growth factor (VEGF) level result came back significantly elevated, at $6,172 \mathrm{pg} / \mathrm{mL}$ (normal range $<600 \mathrm{pg} / \mathrm{mL}$ ). iMCD or TAFRO syndrome was suspected. Further tests showed negative HHV-8 DNA, and her bone marrow biopsy showed signs of myelofibrosis. An axillary lymph node biopsy was performed, and the pathology was consistent with hyaline-vascular type Castleman disease (online suppl. Fig. S2). This patient was finally diagnosed as having TAFRO syndrome (online suppl. Table S2). We increased the steroid dose to methylprednisolone $60 \mathrm{mg} /$ day, and her condition began to improve (Fig. 1, 2).

A regimen of bortezomib $1.3 \mathrm{mg} / \mathrm{m}^{2}$, cyclophosphamide $300 \mathrm{mg} / \mathrm{m}^{2}$, and dexamethasone $40 \mathrm{mg}$ (BCD regimen) every week was used to treat this patient. Prednisone $50 \mathrm{mg} /$ day was given between BCD dosing from April, and then tapered every week thereafter. The patient reported no symptoms and normal self-care ability 1 month later. Until early October, she had a normal PLT and renal function as well as proteinuria $<0.5 \mathrm{~g} / \mathrm{day}$. The inflammatory markers including high-sensitivity (hs)CRP and IL- 6 were suppressed (online suppl. Table S1; Fig. 2). The pleural effusion, pericardial effusion, ascites, and splenomegaly on CT scan also demonstrated continuous improvements (Fig. 1). The patient tolerated this therapy well. She had only mild myelosuppression (a minimum white blood cell count of $2.74 \times 10^{9} / \mathrm{L}$, minimum $\mathrm{Hb} 94 \mathrm{~g} / \mathrm{L}$, and minimum PLT $69 \times 10^{9} / \mathrm{L}$ ) presumably secondary to cyclophosphamide, which could recover within 1 week after temporary stoppage of BCD. 


\section{Kidney \\ Blood Pressure \\ Research}

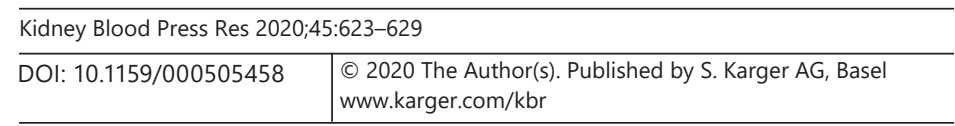

Xia et al.: Acute Kidney Injury Caused by TAFRO Syndrome

\section{Discussion}

TAFRO syndrome is a rare subtype of iMCD with $<100$ cases reported until now. Noriko Iwaki et al. [2] proposed a diagnostic criterion requiring histologic findings from a lymph node biopsy specimen that are compatible with TAFRO syndrome as well as at least 3 TAFRO symptoms, an absence of hypergammaglobulinemia, and small-volume lymphadenopathy. In addition, patients should fulfil at least 1 of the minor criteria: hyperplasia/normoplasia of megakaryocytes in the bone marrow and a high serum ALP but no elevated serum transaminase. However, TAFRO symptoms are not universally present in every patient, making the diagnosis difficult. The multidisciplinary team in our hospital confirmed that this case met all the major and minor criteria for a diagnosis of TAFRO syndrome (online suppl. Table S2).

Renal involvement in TAFRO syndrome is reported in about $55 \%$ of cases [7]. Based on case series published, these patients usually had a low level of proteinuria, microscopic hematuria, elevated $\mathrm{sCr}(0.88-2.28 \mathrm{mg} / \mathrm{dL})$, and oliguria or anuria $[3,4]$. Temporary renal replacement is often needed to correct the volume overload, electrolytes, and acid-base disorder before initiating targeted therapy. Our patient possibly had the highest $\mathrm{s} C \mathrm{r}$ so far reported for cases of TAFRO, indicating her severe disease activity. She may also have had superimposed acute tubular necrosis due to the intravascular volume depletion caused by fluid accumulation in the third space, fever, and poor intake related to nonspecific inflammation. Supportive treatment including hemodialysis was important to stabilize the patient. Fortunately, she gained full recovery of her renal function after steroids and subsequent BCD treatment.

Until 2019, 21 cases of TAFRO syndrome received renal biopsy worldwide. The 2 patterns described are most consistent with mesangial proliferative glomerular nephritis (MPGN) (42\%) and a thrombotic microangiopathy (TMA)-like histology (58\%), but without fibrin thrombi in the glomerular capillaries or arterioles [8]. Some of the MPGN-like patients had glomerulocapillary deposits while others showed weak or negative immunofluorescence results. We did not perform renal biopsy in this patient because of her severe thrombocytopenia and also her complete and rapid recovery of renal function. Based on the clinical pattern of her renal involvement, it is presumed that we could see similar TMA-like or MPGN-like histology in this patient. Acute tubular injury or even acute tubular necrosis might have been superimposed as well. However, renal pathology is not decisive in diagnosing TAFRO syndrome.

The pathogenesis of acute oliguric renal failure in TAFRO syndrome remains unclear [3, 8-11]. Based on pathologic findings such as endothelial swelling, double contours of GBM, and thickening of the basement membrane, it is presumed that glomerular endothelial injuries could play a central role in the development of acute renal failure $[3,8]$. There are various types of renal histology reported in Castleman disease including AA-type amyloidosis, TMA, focal segmental glomerular sclerosis, interstitial nephritis, and even crescentic glomerulonephritis $[12,13]$. We note that another hematologic disorder called polyneuropathy, organomegaly, endocrinopathy, monoclonal gammopathy, and skin changes (POEMS) syndrome has a very similar renal histology pattern to that of TAFRO patients [14]. Interestingly, patients with TAFRO syndrome, Castleman's disease patients demonstrating TMA-like lesions, and POEMS patients share the common disorder of a significantly elevated serum VEGF level [8, $14,15]$. It is speculated that high circulating levels of IL- 6 and VEGF downregulate glomerular VEGF by podocytes via a negative-feedback mechanism, leading to the loss of glomerular endothelial cell fenestrations, just like those observed in patients receiving anti-VEGF agents and in those with pre-eclampsia $[13,16]$. It is well known that sFlt1 protein, a splice variant of the VEGF receptor Flt-1 or VEGF receptor 1, is a circulating antiangiogenic protein that inhibits VEGF signaling in pre-eclampsia patients $[17,18]$. We hypothesize that certain factor such as sFlt-1 might block the VEGF receptor in the target organ, causing this paradoxical phenomenon of VEGF being elevated in the serum but depleted in the tissues. 


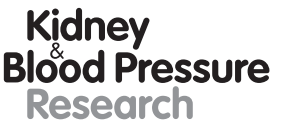

Kidney
Blood Pressure Research

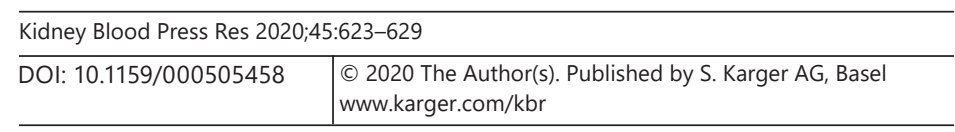

Xia et al.: Acute Kidney Injury Caused by TAFRO Syndrome

Due to a scarcity of cases and a lack of understanding of the disease mechanisms, there is no standard treatment for TAFRO syndrome. The main therapeutic options include corticosteroids, immunosuppressive therapy, rituximab or rituximab-based therapy, anti-IL-6 therapies (tocilizumab and siltuximab), thalidomide combined with cyclophosphamide and prednisone, and plasma exchange [3, 5, 6, 9, 10,19-22]. The Japanese TAFRO Research Group recommends high-dose steroids, tocilizumab, and cyclosporine A for TAFRO patients [7]. However, based on the data reviewed by the international, evidence-based consensus treatment guidelines for iMCD, the rate of treatment failure varies from 29 to $100 \%$ [6]. The mortality of this group of patients could be 11.1-12.0\%, secondary to disease progression or severe infection [2,7]. A drastic inflammatory process in the early disease phase was the major problem in our patient, and this would partially explain her initial response to the highdose steroids. We tried to treat this patient with a weekly BCD regimen with medium-dose prednisone in between to suppress the ongoing inflammation. After 4 months, we succeeded in tapering the prednisone to $5 \mathrm{mg} /$ day and the patient is currently symptom-free. To our knowledge, this case might be the first TAFRO patient successfully treated with this regimen.

In conclusion, we have presented a rare case of acute anuric renal failure caused by TAFRO syndrome. Elevated VEGF may be a useful marker, and lymph node pathology compatible with Castleman disease is critical to the final diagnosis. The weekly BCD regimen with a medium dose of prednisone in between provides a potential therapeutic option for treating this group of patients.

\section{Statement of Ethics}

Written informed consent was obtained from the patient.

\section{Disclosure Statement}

The authors declare that they have no conflict of interests.

\section{Funding Sources}

This work was supported in part by funding from the National Natural Science Foundation of China (81470937 and 81641024), the Beijing Municipal Science and Technology ProjectCapital Specialized Clinical Application Project (Z171100001017196), the Key Research and Development Program of Ningxia (2018BFG02010), the Chinese Academy of Medical Sciences Innovation Fund for Medical Sciences (2016-I2M-2-004), the National Key Research and Development Program of China (2016YFC0901500), and the Xiehe Scholarship Grant to L.C. X.P. was supported by Fundamental Research Funds for the Central Universities (3332019029).

\section{Author Contributions}

X.P., L.Z., M.Z., T.Z., R.L., and L.C. managed the patient in hospital. J.W. made the pathologic diagnosis and provided the pathologic pictures. J.L. and L.Z. supported the test of serum VEGF level and proposed the weekly BCD regimen. Y.Q., L.C., and X.L. approved and supervised the BCD treatment in the ward. X.P., L.Z., and M.Z. drafted the original manuscript. X.S. and L.C. reviewed and modified the manuscript. All authors approved the paper. 


\section{Kidney \\ Blood Pressure \\ Research}

\begin{tabular}{l|l}
\hline Kidney Blood Press Res 2020;45:623-629 \\
\hline DOI: 10.1159/000505458 & $\begin{array}{l}\text { @ 2020 The Author(s). Published by S. Karger AG, Basel } \\
\text { www.karger.com/kbr }\end{array}$ \\
\hline
\end{tabular}

Xia et al.: Acute Kidney Injury Caused by TAFRO Syndrome

\section{References}

1 Takai K, Nikkuni K, Shibuya H, Hashidate H. Thrombocytopenia with mild bone marrow fibrosis accompanied by fever, pleural effusion, ascites and hepatosplenomegaly. Rinsho Ketsueki. 2010 May;51(5):320-5. Japanese.

2 Iwaki N, Fajgenbaum DC, Nabel CS, Gion Y, Kondo E, Kawano M, et al. Clinicopathologic analysis of TAFRO syndrome demonstrates a distinct subtype of HHV-8-negative multicentric Castleman disease. Am J Hematol. 2016 Feb;91(2):220-6.

3 Tanaka M, Tsujimoto H, Yamamoto K, Shimoda S, Oka K, Takeoka H. Clinicopathological features of progressive renal involvement in TAFRO syndrome: a case report and literature review. Medicine (Baltimore). 2017 Oct; 96(40):e8216.

4 Nishimura Y, Hanayama Y, Fujii N, Kondo E, Otsuka F. Comparison of the Clinical Characteristics of TAFRO Syndrome and Idiopathic Multicentric Castleman Disease in General Internal Medicine: A 6-Year Retrospective Study. Intern Med J. 2020 Feb;50(2):184-91.

5 Igawa T, Sato Y. TAFRO Syndrome. Hematol Oncol Clin North Am. 2018 Feb;32(1):107-18.

6 van Rhee F, Voorhees P, Dispenzieri A, Fosså A, Srkalovic G, Ide M, et al. International, evidence-based consensus treatment guidelines for idiopathic multicentric Castleman disease. Blood. 2018 Nov; 132(20): 2115-24.

7 Masaki Y, Kawabata H, Takai K, Tsukamoto N, Fujimoto S, Ishigaki Y, et al.; Japanese TAFRO syndrome research team. Proposed diagnostic criteria, disease severity classification, and treatment strategy for a novel disorder; TAFRO syndrome. Rinsho Ketsueki. 2016;57(10):2029-37.

8 Leurs A, Gnemmi V, Lionet A, Renaud L, Gibier JB, Copin MC, et al. Renal Pathologic Findings in TAFRO Syndrome: Is There a Continuum between Thrombotic Microangiopathy and Membranoproliferative Glomerulonephritis? A Case Report and Literature Review. Front Immunol. 2019 Jun;10:1489.

9 Shirai T, Onishi A, Waki D, Saegusa J, Morinobu A. Successful treatment with tacrolimus in TAFRO syndrome: two case reports and literature review. Medicine (Baltimore). 2018 Jun;97(23):e11045.

10 Tsurumi H, Fujigaki Y, Yamamoto T, Iino R, Taniguchi K, Nagura M, et al. Remission of Refractory Ascites and Discontinuation of Hemodialysis after Additional Rituximab to Long-term Glucocorticoid Therapy in a Patient with TAFRO Syndrome. Intern Med. 2018 May;57(10):1433-8.

11 Mizuno H, Sekine A, Oguro M, Oshima Y, Kawada M, Sumida K, et al. Renal histology in a patient with TAFRO syndrome: a case report. Hum Pathol. 2018 Dec;82:258-63.

12 Xu D, Lv J, Dong Y, Wang S, Su T, Zhou F, et al. Renal involvement in a large cohort of Chinese patients with Castleman disease. Nephrol Dial Transplant. 2012 Oct;27 Suppl 3:iii119-25.

13 El Karoui K, Vuiblet V, Dion D, Izzedine H, Guitard J, Frimat L, et al. Renal involvement in Castleman disease. Nephrol Dial Transplant. 2011 Feb;26(2):599-609.

14 Ye W, Wang C, Cai QQ, Cai H, Duan MH, Li H, et al. Renal impairment in patients with polyneuropathy, organomegaly, endocrinopathy, monoclonal gammopathy and skin changes syndrome: incidence, treatment and outcome. Nephrol Dial Transplant. 2016 Feb;31(2):275-83.

15 Sun PP, Yu XJ, Wang SX, Zhou XJ, Qu L, Zhang F, et al. Association of vascular endothelial growth factor and renal thrombotic microangiopathy-like lesions in patients with Castleman's disease. Nephrology (Carlton). 2020 Feb;25(2):125-34.

16 Eremina V, Jefferson JA, Kowalewska J, Hochster H, Haas M, Weisstuch J, et al. VEGF inhibition and renal thrombotic microangiopathy. N Engl J Med. 2008 Mar;358(11):1129-36.

17 Lemoine E, Thadhani R. Affordable Preeclampsia Therapeutics. Trends Pharmacol Sci. 2019 Feb;40(2):85-7.

18 Karumanchi SA. Angiogenic Factors in Preeclampsia: From Diagnosis to Therapy. Hypertension. 2016 Jun; 67(6):1072-9.

19 Meguri Y, Asada N, Nakasako Y, Kondo E, Kambara Y, Yamamoto A, et al. A case report of TAFRO syndrome successfully treated by immunosuppressive therapies with plasma exchange. Ann Hematol. 2019 Feb;98(2): 537-9.

20 Zhang L, Zhao AL, Duan MH, Li ZY, Cao XX, Feng J, et al. Phase 2 study using oral thalidomide-cyclophosphamide-prednisone for idiopathic multicentric Castleman disease. Blood. 2019 Apr;133(16):1720-8.

21 Noda Y, Saka Y, Kato A, Mimura T, Naruse T. Successful rituximab treatment of TAFRO syndrome with pathological findings of glomerular endothelial damage. Clin Nephrol Case Stud. 2018 Jun;6(01):16-20.

22 Ito S, Uchida T, Itai H, Yamashiro A, Yamagata A, Matsubara H, et al. Serial Manifestation of Acute Kidney Injury and Nephrotic Syndrome in a Patient with TAFRO Syndrome. Intern Med. 2018 Nov;57(21):3129-33. 